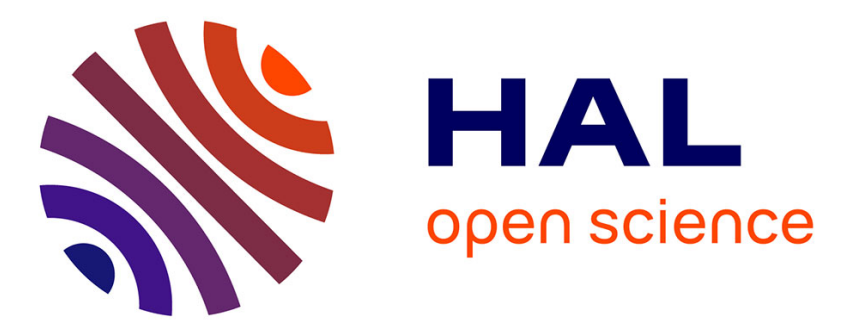

\title{
Rapid typing of neonatal isolates using polymerase chain reaction for repeat regions in surface protein genes
}

\author{
A. Ohlin, A. Bäckman, B. Söderquist, S. Wingren, M. Björkqvist
}

\section{To cite this version:}

A. Ohlin, A. Bäckman, B. Söderquist, S. Wingren, M. Björkqvist. Rapid typing of neonatal isolates using polymerase chain reaction for repeat regions in surface protein genes. European Journal of Clinical Microbiology and Infectious Diseases, 2010, 29 (6), pp.699-704. 10.1007/s10096-010-0917-z . hal-00584832

\section{HAL Id: hal-00584832 \\ https://hal.science/hal-00584832}

Submitted on 11 Apr 2011

HAL is a multi-disciplinary open access archive for the deposit and dissemination of scientific research documents, whether they are published or not. The documents may come from teaching and research institutions in France or abroad, or from public or private research centers.
L'archive ouverte pluridisciplinaire HAL, est destinée au dépôt et à la diffusion de documents scientifiques de niveau recherche, publiés ou non, émanant des établissements d'enseignement et de recherche français ou étrangers, des laboratoires publics ou privés. 
Diseases

Editorial Manager(tm) for European Journal of Clinical Microbiology \& Infectious

Manuscript Draft

Manuscript Number: EJCMID-D-10-00040

Title: Rapid typing of neonatal Staphylococcus epidermidis isolates using PCR for repeat regions in surface protein genes

Article Type: Article

Keywords: 1; Staphylococcus epidermidis 2; genotype 3; PCR 4; human

Corresponding Author: Dr Andreas Ohlin,

Corresponding Author's Institution:

First Author: Andreas Ohlin

Order of Authors: Andreas Ohlin; Anders Bäckman; Bo Söderquist; Sten Wingren; Maria Björkqvist

Abstract: Abstract

Purpose. Staphylococcus epidermidis is a significant pathogen in neonatal sepsis and other nosocomial infections. For further investigations of the colonisation patterns and invasive pathways typing methods that are applicable on large populations of bacterial isolates are warranted.

Methods. In the present study a genotyping method based on polymerase chain reaction (PCR) for the repeat regions of four genes (sdrG, sdrF, aap and sesE) that encode for bacterial surface proteins was developed and applied to a sample of well-characterised neonatal blood isolates of S. epidermidis $(\mathrm{n}=$ 49). The PCR products were visualised on agarose gel (sdrG, sdrF and ses E) or by fragment analysis (aap). The discriminatory index (D-index) for genotyping of the different genes was compared to genotyping by pulsed-field gel electrophoresis.

Results. The highest D-index for the PCR-based typing methods was found for the combination of sdrF, sdrG, and aap (D-index 0.94), whereas the optimal two-gene combination (sdrF and aap) resulted in a D-index of 0.92.

Conclusions. We conclude that the described method can be used for genotyping of large populations of S. epidermidis isolates with a sufficient discriminatory capacity, and we suggest that the combination of sdrF and aap is most suitable to use.

Suggested Reviewers: Gordon L Archer

Division of infectious diseases, Department of internal medicine, Virginia commonwealth university garcher@vcu.edu

Gordon L Archer is the corresponding author of one of the most important references in this article.

Richard V Goering

Department of Medical Microbiology and Immunology, Creighton University School of Medicine, rgoeri@creighton.edu

Richard V Groening has shown a great interest in typing of Staphylocci and has also published several articles on the subject. 



\title{
Rapid typing of neonatal Staphylococcus epidermidis isolates using PCR for repeat regions in surface protein genes
}

\author{
Andreas Ohlin $^{1 *}$, Anders Bäckman ${ }^{2}$, Bo Söderquist ${ }^{3}$, Sten Wingren ${ }^{4}$, Maria Björkqvist ${ }^{1,4}$ \\ ${ }^{1}$ Department of Paediatrics, ${ }^{2}$ Clinical Research Centre, and ${ }^{3}$ Department of Clinical \\ Microbiology, Örebro University Hospital, Örebro, Sweden, ${ }^{4}$ School of Health and Medical \\ Sciences, Örebro University, Örebro, Sweden
}

*Corresponding Author:

Andreas Ohlin

andreas.ohlin@orebroll.se

Department of Paediatrics, Örebro University Hospital

70185 Örebro

Sweden

$+46736137200$

Running title: PCR-based typing of neonatal Staphylococcus epidermidis

Key words: Staphylococcus epidermidis, genotype, PCR, human 

warranted.

Methods. In the present study a genotyping method based on polymerase chain reaction (PCR) for the repeat regions of four genes ( $s d r G, s d r F$, aap and $s e s E$ ) that encode for bacterial surface proteins was developed and applied to a sample of well-characterised neonatal blood isolates of $S$. epidermidis $(\mathrm{n}=49)$. The PCR products were visualised on agarose gel (s $d r G, \mathrm{~s} d r F$ and ses $E$ ) or by fragment analysis (aap). The discriminatory index (D-index) for genotyping of the different genes was compared to genotyping by pulsed-field gel electrophoresis.

Results. The highest D-index for the PCR-based typing methods was found for the combination of $\mathrm{s} d r F, \mathrm{~s} d r G$, and aap (D-index 0.94), whereas the optimal two-gene combination ( $\mathrm{s} d r F$ and aap) resulted in a D-index of 0.92 .

Conclusions. We conclude that the described method can be used for genotyping of large populations of $S$. epidermidis isolates with a sufficient discriminatory capacity, and we suggest that the combination of $s d r F$ and aap is most suitable to use.

\section{Introduction}

Neonatal sepsis is a common complication in neonatal intensive care. Premature birth, low birth weight, and long hospital stay are significant risk factors for this condition [1]. In parallel with the survival of more premature and vulnerable infants, S. epidermidis, which is known as a commensal, has emerged as the most common cause of neonatal sepsis [2]. These 
infections are often caused by multiresistant strains that can persist in neonatal intensive care units (NICUs) for long periods [3-9].

To prevent the spreading of these strains, additional knowledge of the factors that influence the colonisation of newborns, the invasive routes, and other pathogenetic factors of $S$. epidermidis is warranted. These issues cannot be investigated without manageable typing methods requiring an acceptable workload that can fingerprint the large numbers of $S$. epidermidis isolates.

In the search for typing tools applicable to large samples of bacterial isolates, sequencing of the repeat regions in the genes encoding S. epidermidis surface proteins, such as aap and $s d r G$, has been suggested as a novel typing method [10]. The aim of the present study was to develop a method that combines the diversity in the serine-aspartate dipeptide repeats with an easy-to-use detection approach, and to apply this method to a well-characterised collection of neonatal blood isolates, thus enabling comparison with pulsed-field gel electrophoresis (PFGE).

\section{Materials and Methods}

\section{Bacterial isolates}

Fifty clinical isolates of $S$. epidermidis previously determined to species level by Staph-zym (Rosco Diagnostica, Taastrup, Denmark), obtained from blood cultures of neonates and collected from 1990 to 1997 at the Neonatal Intensive Care Unit, Örebro University Hospital, were included in this study. After subcultivation, one of the bacterial isolates was identified as a $S$. hominis by sequencing of the $r p o B$ gene [11] and was hence excluded, leaving the remaining 49 S. epidermidis isolates for further analysis. These isolates have previously been characterised phenotypically and genotypically, including PFGE [4]. According to the clinical and laboratory criteria applied in the former study, 11 isolates were classified as representing 
true neonatal sepsis and 38 isolates were considered as contaminants. All isolates were stored at $-70^{\circ} \mathrm{C}$ pending analysis and were subcultured on blood agar plates $(4.25 \%$ Columbia II Agar, BBL, Becton Dickinson, MD, USA; 0.3\% Agar No. 2, Lab M Ltd., Bury, UK; 5\% bovine blood) and checked for purity prior to DNA preparation.

\section{Pulsed-field gel electrophoresis}

The isolates were characterised by PFGE of chromosomal SmaI digests, prepared with the GenePath Group 1 Reagent Kit (Bio-Rad Laboratories, Hercules, CA, USA), and these data originated from a previous study [4].

\section{DNA preparation}

A QIAamp DNA Mini Kit was used to isolate DNA from the strains (Protocol D, QIAGEN, Hilden, Germany) with the following modifications; Bacteria obtained from the blood agar plate were dissolved in sterile saline and pelleted, dissolved in $180 \mu \mathrm{L}$ ATL buffer (QIAGEN) with $10 \mu \mathrm{L}$ mutanolysin $10 \mathrm{U} / \mu \mathrm{L}$ (Sigma, St. Louis, MO, USA) added and incubated at $37^{\circ} \mathrm{C}$ for $30 \mathrm{~min}$. The mix was treated with proteinase $\mathrm{K}$ and $\mathrm{AL}$ buffer (QIAGEN) at $56^{\circ} \mathrm{C}$ for 30 min and DNA-purified according to the tissue protocol, using ethanol and wash buffers supplied in the kit, and finally eluted in $50 \mu \mathrm{L}$ AE buffer (QIAGEN) and stored at $4^{\circ} \mathrm{C}$.

\section{$P C R$}

The repeat regions for aap and $s d r G$ were amplified by PCR, as previously described by Monk and Archer [10], using Taq PCR Master Mix Kit (QIAGEN, Hilden, Germany) ( $25 \mu \mathrm{L} / 50 \mu \mathrm{L}$ reaction), $2 \mu \mathrm{M}$ of each primer (Applied Biosystems, Warrington, UK) and $2 \mu \mathrm{L}$ DNA preparation. The primers for $s d r F$ and $\operatorname{ses} E$ (Scandinavian Gene Synthesis AB, Köping, Sweden) were designed using the PRIMER3 program and compared with published 
sequences on BLAST (Table 1). To facilitate fragment analysis of the products one primer in each pair was purchased labelled with VIC and 6-FAM, respectively.

The $s d r F$, $s d r G$, and $s e s E$ amplicons $(6 \mu \mathrm{L})$ were treated with restriction enzymes for 2 hours. $S d r G$ was treated with HpyCH4III (New England Biolabs Inc. (NEB), Ipswich, MA, USA) $5 \mathrm{U}$ at $37^{\circ} \mathrm{C}, s d r F$ with BtsCI (NEB) $10 \mathrm{U}$ at $50^{\circ} \mathrm{C}$, and $s e s E$ with BstYI (NEB) $5 \mathrm{U}$ at $60^{\circ} \mathrm{C}$, respectively, and aap was analysed without any enzyme treatment. Enzymes were selected using the NEB cutter computer program.

The PCR products and restriction fragments were separated and visualised on a $1.5 \%$ agarose gel (Agarose 1000, $1 \times$ TBE, $100 \mathrm{~V}, 60 \mathrm{~min}$ ) stained with ethidium bromide. The stained bands were compared to a size marker on each gel (100 bp DNA ladder, Gibco BRL, Carlsbad, CA, USA).

The product sizes of all aap PCR products and the enzyme-treated fragments from $s d r F$, $s d r G$, and $\operatorname{ses} E$ that yielded similar visual results were confirmed by fragment analysis, using ABI 310 or ABI 3130xl, and compared to LIZ-1200 (GeneScan 1200 LIZ, ABI, Foster City, CA, USA) size standard in Peakscanner 1.0/GeneMapper 4.0 (ABI).

\section{Statistical methods}

To evaluate discriminatory capacity of different PCR methods, a discrimination index (D-index) for each included gene and for combinations of the studied genes was calculated [13]. Briefly, the D-index represents the likelihood that two randomly picked strains from the sample are separated by the method and recognised as two different strains. This means that a high D-index (close to one) divides the sample into many small groups, whereas, a low D-index (close to zero) indicates that the sample is only divided into a few large groups. Confidence intervals of 95\% were calculated, as described by Grundmann et al. [14]. 


\section{Results}

The $s d r G$, $s d r F$, and $s e s E$ repeats were present in all 49 isolates, whereas aap was detected in $38 / 49$ isolates. When the primary PCR products of $s d r G$, $s d r F$, and $s e s E$ were visualised on the agarose gels, all genes had a single predominating genotype, $s d r G, 35$ isolates; $s d r F, 36$ isolates; and sesE, 44 isolates, respectively, with amplicons so similar in size that they were indistinguishable on the gel. To further enhance the discrimination between these genotypes, the PCR products were treated with restriction enzymes, and the subtypes of isolates with similar band patterns were confirmed by fragment analysis.

For aap the primary PCR products of all bacterial isolates that carried the aap gene were of similar size and thus difficult to separate on the agarose gel. Since no suitable restriction enzyme for the product could be identified, the sizes of these amplicons were instead determined by fragment analysis.

$s d r G$

After treatment with HpyCH4III restriction enzyme, the $s d r G$ samples showed 2-4 distinct bands of a size ranging from 100 to 300 base pairs (bp), representing 13 different genotypes (Table 2). The most common genotype included 25 isolates, which all had identical band patterns. Four genotypes comprised 6, 5, 3, and 2 isolates, respectively, and the final 8 genotypes were only represented by single isolates.

$s d r F$

After treatment with BtsCI restriction enzyme, the $s d r F$ samples showed 1-3 clear distinct bands in the size range of $250-1,000 \mathrm{bp}$ that were used for typing and corresponded to 17 different genotypes. The most common genotype consisted of 19 isolates with identical band 
patterns (Table 2). Five genotypes included 7, 5, 3, 3, and 2 isolates, respectively, and 11 genotypes were only represented by single isolates.

$\operatorname{ses} E$

After treatment with BstYI restriction enzyme, the sesE samples showed 1-5 distinct bands in the size range of 100-700 bp. These were used for typing and resulted in 8 different genotypes (Table 2). Twenty-four isolates constituted the most common genotype, and the others had 12, 4, 3, and 3 strains, respectively. Three genotypes were represented by single isolates.

aap

Since the aap repeat was found in 38/49 isolates, one genotype consisted of 11 isolates without aap. The remaining 38 isolates where analysed with fragment analysis showing a size range of 414-514 bp and 7 genotypes with $13,11,8,2,2,1$, and 1 strains respectively.

\section{$P F G E$}

Six isolates were indistinguishable by PFGE, and 17 were classified as single isolates. The remaining isolates were assigned to various genotypes with 2 or 3 isolates. The 6 indistinguishable isolates were also indistinguishable when analysing $s d r G$, aap and sesE. Regarding $s d r F, 5$ isolates showed an identical result, whereas, one isolate had a different genotype. The 17 single isolates were subdivided into 11 groups with $s d r G$ and $s d r F, 7$ groups with aap, and 6 groups with sesE.

\section{Discriminatory index}

All the calculated D-indices are presented in Table 2. The highest D-index for a single gene was found for $s d r F(0.82)$, followed by aap, $s d r G$, and $s e s E$, respectively. By combining two 
genes, the highest D-index was found in $a a p / s d r F(0.92)$, followed by aap/sdrG. When a third gene was added, the D-index was only marginally enhanced (aap/sdrG/sdrF, $D$-index 0.94$)$. The corresponding figure for PFGE was 0.98.

\section{Correlation between PFGE and surface protein PCR results}

When comparing results of PFGE and the PCR genotyping method, we found concordance in the vast majority of isolates. According to PFGE three clusters including 9, 5 and 5 closely related isolates respectively were identified; the remaining 30 were divided into 5 pairs and 20 single isolates. Using all four of the investigated genes the repeat region PCR characterized the three clusters in exactly the same way as PFGE with the exception of 2 isolates in the largest cluster, where the result for $s d r F$ diverted. One of the clusters including five isolates showed exactly the same genotypes as 2 other isolates that were grouped into one pair and one single isolate according to PFGE. 


\section{Discussion}

In this study we present a novel typing method for S. epidermidis based on PCR and the use of restriction enzymes. The method combines feasibility and low cost with a high discriminatory capacity. We used a gel-based detection in combination with fragment analysis to evaluate the replicates, which enables the method to be applicable to large collections of samples.

S. epidermidis constitutes the predominant bacterial species of the normal skin flora and is represented by numerous isolates per individual but is also a significant nosocomial pathogen in predisposed patients. Nosocomial strains of S. epidermidis regularly display multiple antibiotic resistance, but the emergence of resistance also to glycopeptides during the recent years further impose the importance of epidemiological surveillance $[15,16]$. Thus there is an urgent demand for swift, easy-to-use typing methods for S. epidermidis not only for research purposes but also for clinical use, for instance in case of an out-break of multi-resistant bacterial strains in a hospital setting Its our opinion that PCR of repeat regions can become such a tool that can be applied in a situation when an epidemiological survey has collected hundreds of isolates, and there is need to quickly and easily trim down the number of interesting isolates that qualify for further analysis.

In 2007 Monk and Archer [10] suggested sequencing of repeat regions in aap and $\mathrm{s} d r G$ as a typing system for S. epidermidis and showed that there was sufficient variation in these genes to make them suitable candidates for gene-based typing. Sequencing of the repeat regions gives detailed information and facilitates interlaboratory comparisons, but on the other hand, it is complicated, expensive, and time-consuming, which limits its use to small samples. Therefore we instead suggest a gel-based approach to minimize costs and, thus, make the 
method more suitable for epidemiological studies. Presumably because of these protocol

differences, our D-indices for aap and $s d r G$ seem to be lower than those published by Monk and Archer. To counteract this loss of information when sequencing is replaced by a gel-based approach, two additional genes were studied, and by combining these genes, we can now present a gel-based typing method with a discriminatory power that is comparable to the sequence-based method that Monk and Archer described.

In the present study PFGE was used as a reference method. PFGE or multilocus sequence typing (MLST) are widely used for detailed genotyping of S. epidermidis, but are too laborious to be applicable to large samples of bacterial strains $[3,6,10,17,18]$. The high correlation between PFGE and repeat region PCR found in this study might indicate that the methods share a common biological background. On the basis of our data we suggest that the PCR-based typing method presented in this article can be used as an alternative to PFGE or MLST, when high speed and low costs are a higher priority than maximum discriminatory capacity.

We targeted repeat regions in four genes encoding different cell-wall-anchored proteins. These proteins have been suggested as important factors in the attachment and interaction between bacteria and human cells. There are additional data supporting the view that some of these proteins are expressed during clinical infections with $S$. epidermidis $[12,19]$. It has been suggested that repeats are a way to form coil-like structures that enable the protein to span the cell wall (SD-repeats), or to project the A region of the protein away from the cell wall [12]. Our choice of genes was based on the fact that they had repeat regions with a high rate of variation, as well as a proven stability when recultured [10]. The genes have also been reported to have a high prevalence in sepsis strains, contaminants and skin flora $[12,20,21]$. In our material $78 \%$ of the isolates were positive for aap and $100 \%$ for the other genes. 
We evaluated four different genes and, as presented in Table 2, no single repeat analysis had a satisfactory discriminatory power. Therefore, we also studied combinations of genes and, in our opinion, two genes were sufficient in this sample to achieve what we consider to be an acceptable discriminatory capacity. Adding a third gene only marginally increased the discriminatory capacity. We, therefore, suggest the combination of aap and $s d r F$ as suitable for genotypic typing of neonatal S. epidermidis isolates, but before the generalisation of these results can be stated, other series of S. epidermidis strains with a more heterogeneous origin should evaluated.

\section{Conclusion}

We present a novel gel-based typing method for S. epidermidis based on PCR of four genes encoding cell-wall proteins and the use of restriction enzymes. The method combines feasibility and low cost with a high discriminatory capacity, comparable to that of PFGE and sequence-based methods. In our study a combination of two genes, aap and $s d r F$, was found to be optimal, but other combinations of the four studied genes could be more suitable when applied to another material of S. epidermidis isolates. Our proposed method could be used for epidemiological investigations, for example in case of outbreaks of multiresistant strains or exploring the dynamics of the colonization pattern of newborns. In addition, it enables pathogenic studies of, for example, tracking invasive routes of clinical isolates as well as identification/recognition of virulent clones. 


\section{Acknowledgements}

We would like to thank Anders Magnusson for statistical support and Bengt Hellmark for $r p o B$ sequencing. This work was supported by Örebro University and the Research Committee of Örebro University Hospital. 


\section{References}

1. Healy CM, Palazzi DL, Edwards MS, Campbell JR, Baker CJ (2004) Features of invasive staphylococcal disease in neonates. Pediatrics;114(4):953-961.

2. Bizzarro MJ, Raskind C, Baltimore RS, Gallagher PG (2005) Seventy-five years of neonatal sepsis at Yale: 1928-2003. Pediatrics;116(3):595-602.

3. Klingenberg C, Ronnestad A, Anderson AS, Abrahamsen TG, Zorman J, Villaruz A, et al. (2007) Persistent strains of coagulase-negative staphylococci in a neonatal intensive care unit: virulence factors and invasiveness. Clin Microbiol Infect;13(11):1100-1111.

4. Bjorkqvist M, Soderquist B, Tornqvist E, Sjoberg L, Fredlund H, Kuhn I, et al. (2002) Phenotypic and genotypic characterisation of blood isolates of coagulase-negative staphylococci in the newborn. APMIS;110(4):332-339.

5. Vermont CL, Hartwig NG, Fleer A, de Man P, Verbrugh H, van den Anker J, et al. (1998) Persistence of clones of coagulase-negative staphylococci among premature neonates in neonatal intensive care units: two-center study of bacterial genotyping and patient risk factors. J Clin Microbiol;36(9):2485-2490.

6. Villari P, Sarnataro C, Iacuzio L (2000) Molecular epidemiology of Staphylococcus epidermidis in a neonatal intensive care unit over a three-year period. J Clin Microbiol;38(5):1740-1746.

7. Hira V, Sluijter M, Estevao S, Horst-Kreft D, Ott A, de Groot R, et al. (2007) Clinical and molecular epidemiologic characteristics of coagulase-negative staphylococcal bloodstream infections in intensive care neonates. Pediatr Infect Dis J;26(7):607-612.

8. Widerstrom M, Monsen T, Karlsson C, Wistrom J (2006) Molecular epidemiology of meticillin-resistant coagulase-negative staphylococci in a Swedish county hospital: evidence of intra- and interhospital clonal spread. J Hosp Infect;64(2):177-183. 
9. Monsen T, Karlsson C, Wistrom J (2005) Spread of clones of multidrug-resistant, coagulase-negative staphylococci within a university hospital. Infect Control Hosp Epidemiol;26(1):76-80.

10. Monk AB, Archer GL (2007) Use of outer surface protein repeat regions for improved genotyping of Staphylococcus epidermidis. J Clin Microbiol;45(3):730-735.

11. Hellmark B, Soderquist B, Unemo M (2009) Simultaneous species identification and detection of rifampicin resistance in staphylococci by sequencing of the rpoB gene. Eur J Clin Microbiol Infect Dis;28(2):183-190.

12. Bowden MG, Chen W, Singvall J, Xu Y, Peacock SJ, Valtulina V, et al. (2005) Identification and preliminary characterization of cell-wall-anchored proteins of Staphylococcus epidermidis. Microbiology;151(Pt 5):1453-1464.

13. Hunter PR, Gaston MA (1988) Numerical index of the discriminatory ability of typing systems: an application of Simpson's index of diversity. J Clin Microbiol;26(11):24652466.

14. Grundmann H, Hori S, Tanner G (2001) Determining confidence intervals when measuring genetic diversity and the discriminatory abilities of typing methods for microorganisms. J Clin Microbiol;39(11):4190-4192.

15. Biavasco F, Vignaroli C, Varaldo PE (2000) Glycopeptide resistance in coagulasenegative staphylococci. Eur J Clin Microbiol Infect Dis;19(6):403-417.

16. Center KJ, Reboli AC, Hubler R, Rodgers GL, Long SS (2003) Decreased vancomycin susceptibility of coagulase-negative staphylococci in a neonatal intensive care unit: evidence of spread of Staphylococcus warneri. J Clin Microbiol;41(10):4660-4665.

17. Sivadon V, Rottman M, Quincampoix JC, Prunier E, de Mazancourt P, Bernard L, et al. (2006) Polymorphism of the cell wall-anchoring domain of the autolysin-adhesin 
AtlE and its relationship to sequence type, as revealed by multilocus sequence typing of invasive and commensal Staphylococcus epidermidis strains. J Clin Microbiol;44(5):1839-1843.

18. Thomas JC, Vargas MR, Miragaia M, Peacock SJ, Archer GL, Enright MC (2007) Improved multilocus sequence typing scheme for Staphylococcus epidermidis. J Clin Microbiol;45(2):616-619.

19. McCrea KW, Hartford O, Davis S, Eidhin DN, Lina G, Speziale P, et al. (2000) The serine-aspartate repeat (Sdr) protein family in Staphylococcus epidermidis. Microbiology;146 ( Pt 7):1535-1546.

20. Hartford O, O'Brien L, Schofield K, Wells J, Foster TJ (2001) The Fbe (SdrG) protein of Staphylococcus epidermidis HB promotes bacterial adherence to fibrinogen. Microbiology;147(Pt 9):2545-2552.

21. Nilsson M, Frykberg L, Flock JI, Pei L, Lindberg M, Guss B (1998) A fibrinogenbinding protein of Staphylococcus epidermidis. Infect Immun;66(6):2666-2673. 


\section{Table 1.}

Details of the 4 PCR methods used to study the 49 S. epidermidis isolates in this study.

\begin{tabular}{|c|c|c|c|c|}
\hline Gene & Type of repeat* & Primers & $\begin{array}{c}\text { Restriction } \\
\text { enzyme }\end{array}$ & $\begin{array}{c}\text { Fragment size } \\
\text { Range } \\
\text { (approx) }\end{array}$ \\
\hline aap & B & {$[10]$} & - & $414-514 \mathrm{bp}$ \\
\hline$s d r G$ & serine-aspartate & {$[10]$} & HруCH4III & $700-1200 \mathrm{bp}$ \\
\hline$s d r F$ & serine-aspartate & $\begin{array}{l}\text { SDRF1 (5'-CTGATGGGGAAGATGTTCGT-3') } \\
\text { SDRF2 (5-'TCATGATCTTCATTTGCTCCTG-3') }\end{array}$ & BtsCI & 250-1200 bp \\
\hline sesE & B & $\begin{array}{l}\text { SE1L (5'-CATCTTTATCTGTACCGCCTGT-3') } \\
\text { SE1R (5'-ATCCAACTGCCACTGAACCT-3). }\end{array}$ & BstYI & 800-1200 bp \\
\hline
\end{tabular}

* B repeat - repeat region following the A (ligand) region

Serine-aspartate repeat - repeat region consisting of serine and aspartate that is located downstram from the B-region. Nomenclature adapted from Bowden et al. [12] 
Table 2.

Number of genotypes, and discrimination index (D-index) for the included repeats and combinations of repeats in the included S. epidermidis neonatal blood isolates $(\mathrm{n}=49)$.

\begin{tabular}{|c|c|c|c|c|}
\hline Gene & $\begin{array}{l}\text { Number of } \\
\text { genotypes }\end{array}$ & $\begin{array}{l}\text { No. of isolates with } \\
\text { most common } \\
\text { genotype }(\%)\end{array}$ & D-index & $\begin{array}{c}95 \% \text { confidence } \\
\text { interval }\end{array}$ \\
\hline aap & 8 & $13(27)$ & 0.81 & $0.77-0.86$ \\
\hline$s d r G$ & 13 & $25(51)$ & 0.72 & $0.59-0.85$ \\
\hline$s d r F$ & 17 & $19(39)$ & 0.82 & $0.73-0.92$ \\
\hline sesE & 8 & $24(49)$ & 0.70 & $0.59-0.80$ \\
\hline aap $s d r G$ & 18 & $13(27)$ & 0.89 & $0.84-0.94$ \\
\hline aap sdrF & 21 & $11(22)$ & 0.92 & $0.87-0.96$ \\
\hline aap sesE & 16 & $11(22)$ & 0.88 & $0.83-0.93$ \\
\hline$s d r G s d r F$ & 20 & $15(31)$ & 0.88 & $0.81-0.95$ \\
\hline $\operatorname{sdn} G \operatorname{ses} E$ & 17 & $21(43)$ & 0.80 & $0.69-0.91$ \\
\hline$s d r F$ sesE & 22 & $15(31)$ & 0.88 & $0.81-0.95$ \\
\hline aap $s d r G s d r F$ & 23 & $8(16)$ & 0.94 & $0.91-0.97$ \\
\hline aap sdrG sesE & 21 & $11(22)$ & 0.91 & $0.87-0.96$ \\
\hline aap sdrF sesE & 24 & $11(22)$ & 0.92 & $0.88-0.97$ \\
\hline$s d r G \operatorname{sdrF} \operatorname{ses} E$ & 22 & $12(24)$ & 0.91 & $0.86-0.96$ \\
\hline PFGE & 34 & $6(12)$ & 0.98 & $0.96-0.99$ \\
\hline
\end{tabular}



Letter to the editor
Click here to download Electronic Supplementary Material: Rapid_letter_jan15.doc

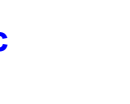

-

(

(2)

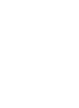

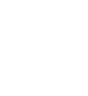

(2)

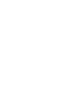

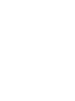
.

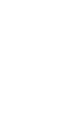
列

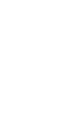
$\sqrt{10}$

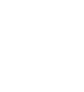

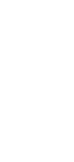

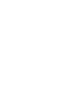
(1)

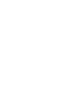

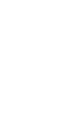

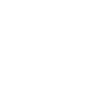
.

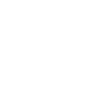
.

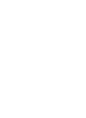

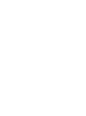
.

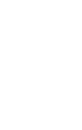

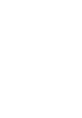

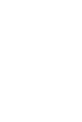

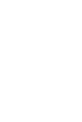
.

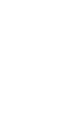

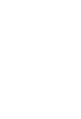

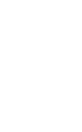

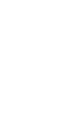
.

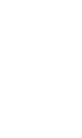

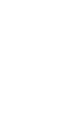

